\title{
Эффективная масса, подвижность носителей заряда и решеточная теплопроводность в нанокомпозитных термоэлектриках на основе халькогенидов висмута и сурьмы
}

\author{
(С) Л.Н. Лукьянова, А.А. Шабалдин, А.Ю. Самунин, О.А. Усов \\ Физико-технический институт им. А.Ф. Иоффе Российской академии наук, \\ 194021 Санкт-Петербург, Россия \\ E-mail: lidia.lukyanova@mail.ioffe.ru
}

Поступила в Редакцию 12 августа 2021 г.

В окончательной редакции 28 августа 2021 г.

Принята к публикации 28 августа 2021 г.

В термоэлектриках $p$-типа на основе халькогенидов висмута и сурьмы с избытком висмута эффективная масса плотности состояний $m / m_{0}$ возрастает в нанокомпозитном и наноструктурированном твердых растворах по сравнению с базовым материалом, полученным методом направленной кристаллизации. Показано, что увеличение $\mathrm{m} / \mathrm{m}_{0}$ связано с ростом эффективного параметра рассеяния $r_{\text {eff }}$ и усилением зависимости времени релаксации от энергии, что характерно для топологических изоляторов. Параметр материала $\beta$, пропорциональный термоэлектрической эффективности $Z T$, при температурах ниже комнатной возрастает сильнее в наноструктурированном составе, чем в нанокомпозите с нановключениями $\mathrm{SiO}_{2}$ вследствие роста $\mathrm{m} / \mathrm{m}_{0}$ и уменьшения решеточной теплопроводности $\kappa_{\mathrm{L}}$. При дальнейшем повышении температуры наибольший рост параметра $\beta$ в базовом материале связан с более высокой подвижностью.

Ключевые слова: халькогениды висмута и сурьмы, наноструктурированные твердые растворы, нанокомпозит, эффективная масса.

DOI: $10.21883 /$ FTP.2021.12.51693.09

\section{1. Введение}

При разработке высокоэффективных объемных термоэлектрических материалов на основе теллурида висмута, относящихся к топологическим изоляторам, в настоящее время большое внимание уделяется наноструктурирированным и нанокомпозитным материалам. В работах [1-3] рассматривается возможность повышения термоэлектрической эффективности в топологических термоэлектриках при более детальном подходе к наноструктурированию, поскольку в ТИ линейная дисперсия и связанная с ней непараболичность зонной структуры, вызванные сильным спин-орбитальным взаимодействием, приводят к увеличению энергетической зависимости спектрального распределения средних длин свободного пробега не только фононов, но и электронов.

Возможность повышения термоэлектрической эффективности в материалах на основе теллурида висмута за счет наноструктурирования объемной матрицы, в которую могут быть добавлены наночастицы металлов, полупроводников или нанокерамик в количестве нескольких весовых процентов от исходной матрицы, рассматривается в работах [4-7]. При такой методике формирования нанокомпозитных термоэлектриков происходит повышение коэффициента Зеебека, связанное с фильтрацией энергии электронов на вновь сформированных интерфейсах за счет добавления наночастиц $[5,7,9]$. Повышение коэффициента Зеебека за счет изменения спектрального распределения длин свободного пробега электронов, когда размеры наноструктурированных зе- рен и включений становятся сравнимыми с длиной свободного пробега электронов, обеспечивает увеличение параметра мощности, компенсируя снижение электропроводности в композитном термоэлектрике.

Однако основное влияние на повышение термоэлектрической эффективности, как правило, происходит за счет снижения решеточной теплопроводности, которое связано с усиленным рассеянием фононов на новых интерфейсах, а именно на границах нанокристаллических зерен и нановключений, а также при рассеянии фононов на дефектах. Наибольшее снижение решеточной теплопроводности наблюдается, когда длина свободного пробега сопоставима с размерами нанокристаллических зерен. Дополнительное снижение полной теплопроводности связано с уменьшением электронной части теплопроводности вследствие снижения электропроводности. Кроме того, снижение электронной теплопроводности и увеличение коэффициента Зеебека может происходить за счет уменьшения влияния биполярной диффузии, как показали теоретические расчеты при анализе транспортных свойств с использованием квантовомеханических неравновесных функций Грина, проведенные в работе [10].

Настоящая работа посвящена исследованию температурных зависимостей эффективной массы плотности состояний $m / m_{0}$, подвижности носителей заряда $\mu_{0}$, решеточной $\kappa_{\mathrm{L}}$ и электронной теплопроводности $\kappa_{e}$ в твердых растворах $\mathrm{Bi}_{2-x+a} \mathrm{Sb}_{x} \mathrm{Te}_{3-b}$ ( $a$ и $b$ определяют отклонение от стехиометриии), полученных горячим прессованием нанопорошков. Рассматривается также влия- 
ние наноструктурирования и включения микрочастиц $\mathrm{SiO}_{2}$ в наноструктурированную матрицу на параметр материала $\beta$, пропорциональный термоэлектрической эффективности.

\section{2. Эффективный параметр рассеяния}

Эффективная масса $m / m_{0}$, подвижность $\mu_{0}$, решеточная $\kappa_{L}$ и электронная теплопроводность $\kappa_{e}$ в наноструктурированных и нанокомпозитных твердых растворах $\mathrm{Bi}_{0.45} \mathrm{Sb}_{1.55} \mathrm{Te}_{2.985}$ были определены из анализа термоэлектрических свойств [11] для интервала температур 80-500 К. Расчеты выполнены для эффективного параметра рассеяния $r_{\text {eff }}$ в модели энергетического спектра с изотропным рассеянием носителей заряда по методике, применявшейся для объемных и пленочных термоэлектриков на основе $\mathrm{Bi}_{2} \mathrm{Te}_{3}$ в работе [12].

Эффективный параметр рассеяния $r_{\text {eff }}$ и приведенный уровень Ферми $\eta$ определяли из решения системы уравнений для коэффициента Зеебека $S\left(r_{\mathrm{eff}}, \eta\right)$ и параметра вырождения $\beta_{d}\left(r_{\mathrm{eff}}, \eta\right)$ методом наименьших квадратов (рис. 1). Выражения для $S$ и $\beta_{d}$ определяли через функции Ферми $\mathrm{F}_{r+n}(\eta)$. В методике определения параметра вырождения $\beta_{d}$ используются данные по гальваномагнитным эффектам, обработанным в многодолинной модели энергетического спектра [12].

Исследования показали, что параметр рассеяния $\left|r_{\text {eff }}\right|$ в выражении энергетической зависимости времени релаксации $\tau=\tau_{0} E^{r}$, где параметр $r=r_{\text {eff }}$ и $\tau_{0}$ не зависит от энергии, и $r_{\text {eff }}$ увеличивается в нанострурированном, особенно в нанокомпозитном материале (рис. 1) по сравнению с базовым твердым раствором. Функции Ферми $\mathrm{F}_{r+n}(\eta)$, где индекс $n=0.5,1.5,2.5$, приведенные на (рис. 2), рассчитывали для соответствующих значений $r_{\text {eff }}$ (рис. 1)

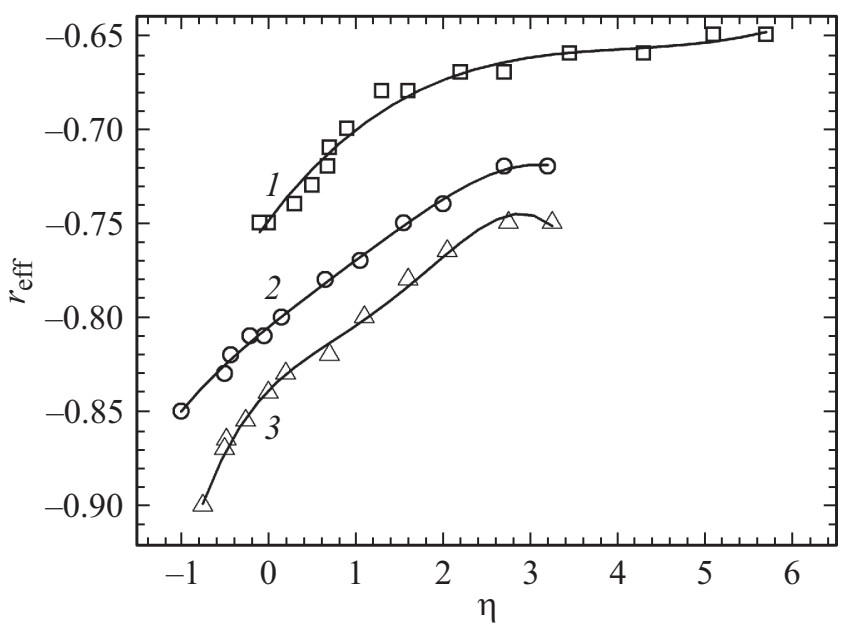

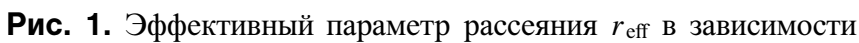
от приведенного уровня Ферми $\eta$ в базовом (1), наноструктурированном (2) и нанокомпозитном (3) твердом растворе $\mathrm{Bi}_{0.45} \mathrm{Sb}_{1.55} \mathrm{Te}_{2.985}$.

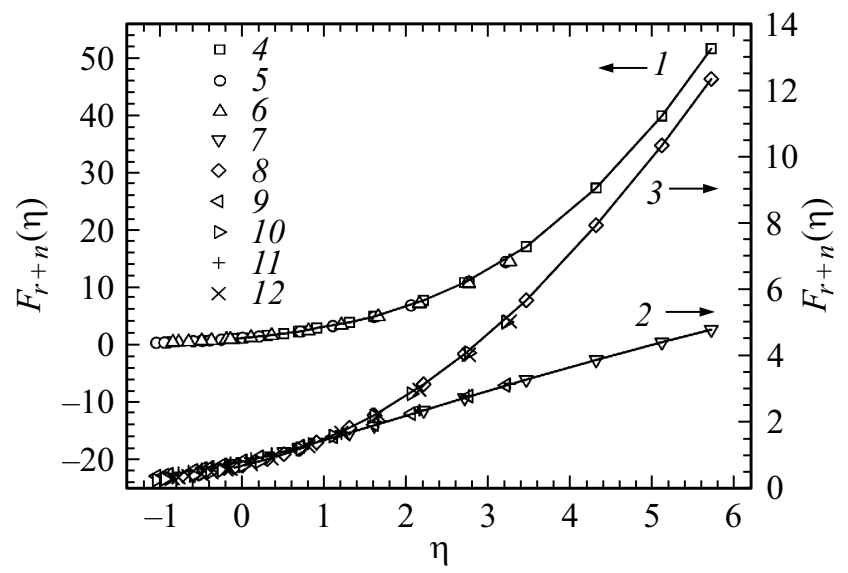

Рис. 2. Функции Ферми $\mathrm{F}_{r+n}(\eta)$, где $n: 1-2.5,2-0.5,3-$ 1.5. 4, 7, 10 - базовый, $5,8,11-$ наноструктурированный и 6, 9, 12 - нанокомпозитный твердый раствор $\mathrm{Bi}_{0.45} \mathrm{Sb}_{1.55} \mathrm{Te}_{2.985}$.

Рост эффективного параметра рассеяния определяет более резкую энергетическую зависимость времени релаксации в исследуемых твердых растворах и является одной из причин эффекта фильтрации носителей заряда при более широком энергетическом спектре электронов, чем фононов в топологических изоляторах, что приводит к росту коэффициента Зеебека [13-16].

\section{3. Эффективная масса и подвижность}

Эффективная масса плотности состояний $m / m_{0}$ возрастает в наноструктурированном и нанокомпозитном материалах по сравнению с базовым твердым раствором во всем исследованном температурном интервале (рис. 3). Наибольшее увеличение $m / m_{0}$ наблюдалось в нанокомпозите, в котором величина $r_{\text {eff }}$ возрастает, что приводит к усилению зависимости времени релаксации от энергии (рис. 1,3, кривые 1 ). Положение максимума на температурной зависимости $m / m_{0}(T)$ в нанокомпозите находится вблизи комнатной температуры и смещается к более высоким температурам: до $320 \mathrm{~K}$ в наноструктурированном составе и до $360 \mathrm{~K}$ в базовом твердом растворе (рис. 3). При дальнейшем росте температуры эффективная масса снижается для всех образцов.

Наиболее высокая подвижность $\mu_{0}$ была получена в базовом твердом растворе. В наноструктурированном составе, особенно в нанокомпозите, величины $\mu_{0}$ снижались, а наклоны температурных зависимостей $\mu_{0}(T)$ ослабевали (рис. 3, кривые 4-6). Такое снижение подвижности $\mu_{0}$ в наноструктурированном и нанокомпозитном материалах связано с рассеянием на объемных примесных дефектах и вследствие увеличения рассеивающих центров за счет границ зерен, а в нанокомпозитном материале с дополнительным рассеянием на границах нановключений. Возможность повышения подвижности связана с коррекцией технологии формирования исследуемых термоэлектриков для получения 


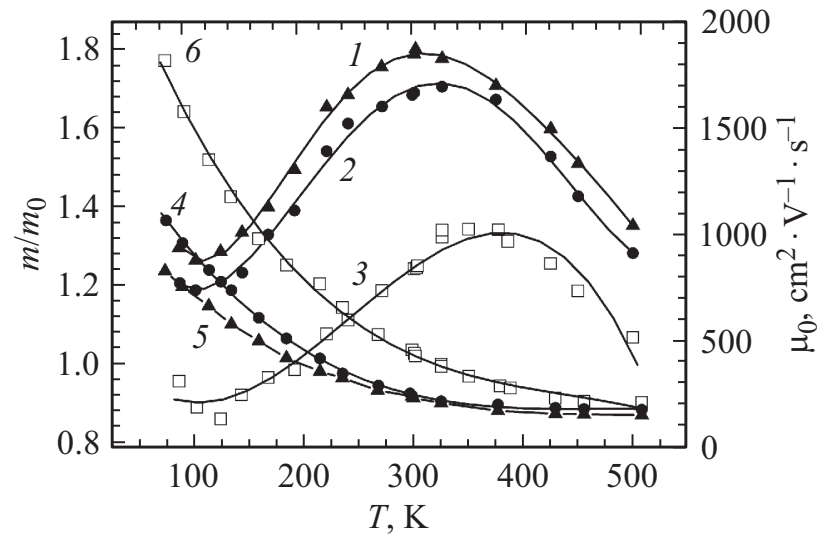

Рис. 3. Температурные зависимости эффективной массы плотности состояний $m / m_{0}(1-3)$ и подвижности носителей заряда $\mu_{0}(4-6)$ в нанокомпозите $(1,4)$, наноструктурированном $(2,5)$ и базовом $(3,6)$ твердом растворе $\mathrm{Bi}_{0.45} \mathrm{Sb}_{1.55} \mathrm{Te}_{2.985}$.

нанозерен и нановключений оптимальных размеров. По оценкам, проведенным в [17], наноструктурирование в ТИ становится наиболее эффективным при размерах зерен в несколько десятков нанометров.

\section{4. Решеточная и электронная теплопроводность. Параметр материала}

Температурные зависимости теплопроводности кристаллической решетки $\kappa_{\mathrm{L}}$ и электронной теплопроводности $\kappa_{e}$ (рис. 4) были определены с учетом $r_{\text {eff }}$ при расчете числа Лоренца $\mathrm{L}\left(r_{\mathrm{eff}}, \eta\right)$ с помощью функций Ферми $\mathrm{F}_{r+n}(\eta)$, (рис. 2).

Как следует из рис. 4, кривые $1-3$, начиная от температур вблизи комнатной и при более высоких температурах, значительно возрастает влияние биполярной диффузии. Поэтому для указанных температур представленные величины на рис. 4 (кривые $1-3$ ) соответствуют сумме $\kappa_{\mathrm{L}}+\kappa_{p n}$, где $\kappa_{p n}-$ вклад в теплопроводность биполярной диффузии.

По сравнению с исходным термоэлектриком величины $\kappa_{\mathrm{L}}$ и $\kappa_{e}$ становятся значительно меньше в наноструктурированном и нанокомпозитном твердых растворах (рис. 4, кривые 5,6). Такое снижение теплопроводности кристаллической решетки $\kappa_{\mathrm{L}}$ в твердых растворах $\mathrm{Bi}_{0.45} \mathrm{Sb}_{1.55} \mathrm{Te}_{2.985}$ связано с интенсивным рассеянием фононов на межфазных и межкристаллитных границах и наноключениях в нанокомпозите.

Уменьшение электронной теплопроводности $\kappa_{e}$ связано со снижением электропроводности и подвижности (рис. 3,4, кривые 4-6). Большее снижение $\kappa_{e}$ и подвижности $\mu_{0}$ наблюдалось в нанокомпозитном твердом растворе (рис. 3,4, кривые 6) в низкотемпературном интервале вплоть до комнатной температуры.
Температурные зависимости $m / m_{0}, \mu_{0}$ и $\kappa_{\mathrm{L}}$ (рис. 3,4$)$ позволяют определить параметр $\beta \propto Z T$ (рис. 5):

$$
\beta=\frac{2(2 \pi)^{3 / 2}}{h^{3} e} k_{0}^{7 / 2}\left(\frac{m}{m_{0}}\right)^{3 / 2} \mu_{0} T^{5 / 2} \kappa_{\mathrm{L}}^{-1} .
$$

При температурах $T<300 \mathrm{~K}$ параметр $\beta$ выше в наноструктурированном твердом растворе (рис. 5, кривая 1) за счет роста эффективной массы $m / m_{0}$ и снижения решеточной теплопроводности $\kappa_{\mathrm{L}}$ (рис. 3,4 , кривые 2), чем в нанокомпозите и базовом материале (рис. 3,4, кривые 1,3). При дальнейшем повышении температуры наибольший рост параметра $\beta$ наблюдается в базовом твердом растворе вследствие более высокой подвижности и небольшого снижения величины $\kappa_{\mathrm{L}}+\kappa_{e}$ при $T>450 \mathrm{~K}$ (рис. 3,4, кривые 1 ). В отличие от низкотемпературной области небольшое увеличение $\beta$ получено при температурах выше комнатной в нанокомпозитном твердом растворе по сравнению с наноструктурированным составом, что связано с ростом эффективной массы $m / m_{0}$, который компенсирует

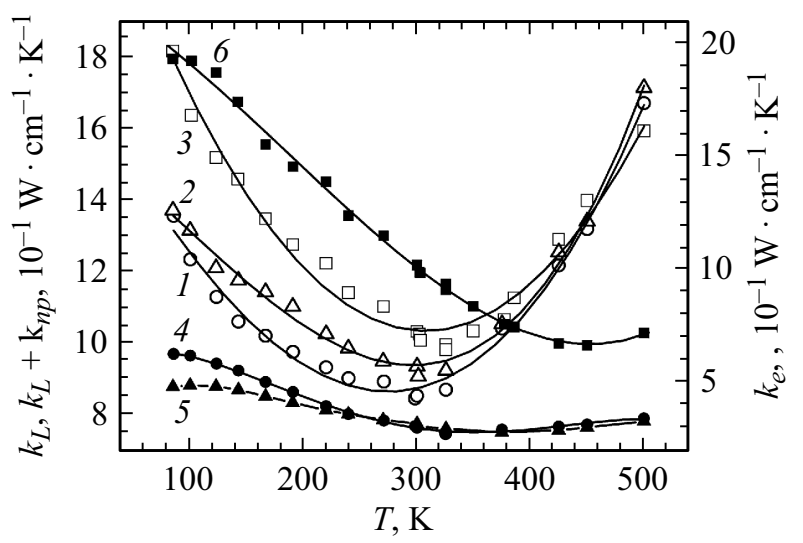

Рис. 4. Температурные зависимости теплопроводности кристаллической решетки $\kappa_{\mathrm{L}}(1-3)$ и электронной теплопроводности $\kappa_{e}(4-6)$ в нанокомпозите $(1,4)$, наноструктурированном $(2,5)$ и базовом $(3,6)$ твердом растворе $\mathrm{Bi}_{0.45} \mathrm{Sb}_{1.55} \mathrm{Te}_{2.985}$.

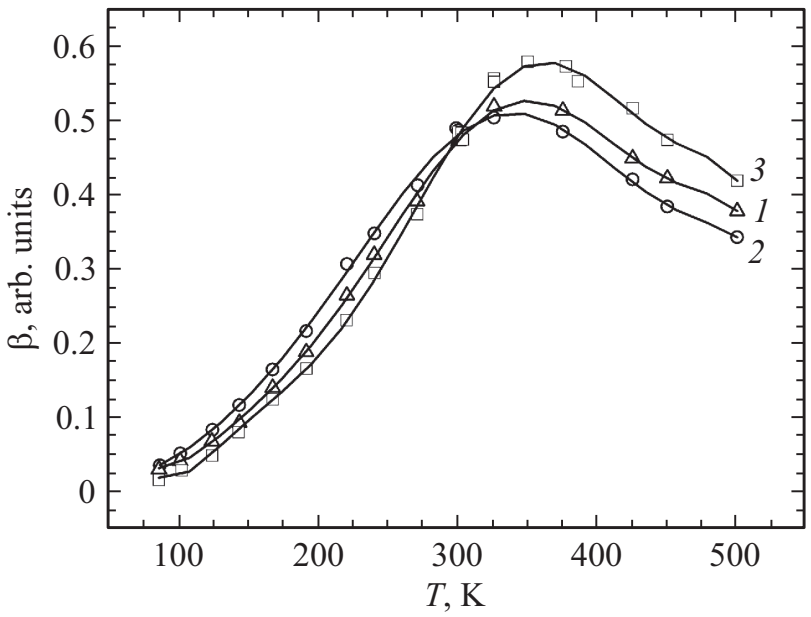

Рис. 5. Температурные зависимости параметра $\beta$ в нанокомпозите (1), наноструктурированном (2) и базовом (3) твердом растворе $\mathrm{Bi}_{0.45} \mathrm{Sb}_{1.55} \mathrm{Te}_{2.985}$. 
увеличение $\kappa_{\mathrm{L}}+\kappa_{e}$ в нанокомпозите (рис. 3, 4, кривые 2,3$)$. Характер поведения параметра $\beta$ согласуется с величинами $Z T$, полученными в твердом растворе $\mathrm{Bi}_{0.45} \mathrm{Sb}_{1.55} \mathrm{Te}_{2.985}$ [11]. В наноструктурированном твердом растворе увеличение $\langle Z T\rangle$ достигает 60\% в интервале температур 80-320 K, а при температурах выше комнатной термоэлектрическая эффективность возрастает в базовом материале, полученном методом направленной кристаллизации и $Z T_{\max } \approx 1$ при $390 \mathrm{~K}$.

\section{5. Заключение}

В результате проведенных исследований в твердых растворах $\mathrm{Bi}_{0.45} \mathrm{Sb}_{1.55} \mathrm{Te}_{2.985}$ были определены эффективная масса плотности состояний $m / m_{0}$, подвижность $\mu_{0}$, решеточная $\kappa_{\mathrm{L}}$ и электронная теплопроводность $\kappa_{e}$ для эффективного параметра рассеяния $r_{\mathrm{eff}}$, определяющего энергетическую зависимость времени релаксации. Показано, что с ростом $r_{\text {eff }}$ зависимость времени релаксации от энергии усиливается в нанокомпозитном и нанострурированном материале по сравнению с базовым твердым раствором.

Рост эффективной массы плотности состояний $m / m_{0}$ в области температур ниже комнатной в термоэлектрике $\mathrm{Bi}_{0.45} \mathrm{Sb}_{1.55} \mathrm{Te}_{2.985}$ вместе со снижением теплопроводности кристаллической решетк $\kappa_{\mathrm{L}}$ приводит к большему увеличению параметра $\beta \propto Z T$ в наноструктурированном материале, чем в нанокомпозите, в котором наблюдается дополнительное снижение подвижности при рассеянии носителей заряда на дефектах, образованных включениями $\mathrm{SiO}_{2}$. При температурах выше комнатной в интервале $300-500 \mathrm{~K}$ небольшое увеличение параметра $\beta$ получено в нанокомпозитном твердом растворе по сравнению с наноструктурированным составом вследствие роста эффективной массы $m / m_{0}$. Однако при высоких температурах наибольший рост параметра $\beta$, связанный с более высокой подвижностью, наблюдается в базовом материале.

\section{Финансирование работы}

Работа выполнена при финансовой поддержке Российского фонда фундаментальных исследований (грант № 20-08-00464).

\section{Конфликт интересов}

Авторы заявляют, что у них нет конфликта интересов.

\section{Список литературы}

[1] T.-H. Liu, J. Zhou, M. Li, Z. Ding, Q. Song, B. Liao, L. Fu, G. Chen. Proc. Natl. Acad. Sci. USA, 115, 879-885 (2018).

[2] N. Xu, Y. Xu, J. Zhu. npj Quant. Mater., 2, 51 (2017)

[3] J.P. Heremans, V. Jovovic, E.S. Toberer, A. Saramat, K. Kurosaki, A. Charoenphakdee, S. Yamanaka, G.J. Snyder. Science, 321, 554 (2008).
[4] W. Xie, J. He, H.J. Kang, X. Tang, S. Zhu, M. Laver, S. Wang, J.R.D. Copley, C.M. Brown, Q. Zhang, T.M. Tritt. Nano Lett., 10, 3283 (2010).

[5] B. Madavali, H.S. Kim, K.H. Lee, S.J. Hong. J. Appl. Phys., 121, 225104 (2017).

[6] L.Q. Tan, J.F. Li, D.W. Liu, F. Li, Z.Y. Li, M. Zou, K. Wang. Adv. Funct. Mater., 23, 4317 (2013).

[7] Y.C. Dou, X.Y. Qin, D. Li, L.L. Li, T.H. Zou, Q.Q. Wang. J. Appl. Phys., 114, 044906 (2013).

[8] S. Seo, K. Lee, Y. Jeong, M.-W. Oh, B. Yoo. J. Phys. Chem. C, 119, 18038 (2015).

[9] T. Zou, X. Qin, Y. Zhang, X. Li, Z. Zeng, D. Li, J. Zhang, H. Xin, W. Xie, A. Weidenkaff. Sci. Rep., 5, 17803 (2015).

[10] S. Foster, N. Neophytou. Comp. Mater. Sci., 164, 91 (2019)

[11] А.А. Шабалдин, П.П. Константинов, Д.А. Курдюков, Л.Н. Лукьянова, А.Ю. Самунин, Е.Ю. Стовпяга, А.Т. Бурков. ФТП, 53, 6, 751 (2019).

[12] L.N. Lukyanova, Yu.A. Boikov, Danilov, O.A. Usov, M.P. Volkov, V.A. Kutasov. Semicond. Sci. Technol., 30, 015011 (2015).

[13] G. Jiang, J. Yi, L. Miao, P. Tang, H. Huang, C. Zhao, S. Wen. Sci. Rep., 8, 2355 (2018).

[14] R.V. Gorbachev, A.K. Geim, M.I. Katsnelson, K.S. Novoselov, T. Tudorovskiy, I.V. Grigorieva, A.H. MacDonald, S.V. Morozov, K. Watanabe, T. Taniguchi, L.A. Ponomarenko. Nature Physics, 8, 896 (2012).

[15] D. Baldomir, D. Failde. Sci. Rep., 9, 6324 (2019).

[16] M.K. Jacobsen, R.S. Kumar, A.L. Cornelius, S.V. Sinogeiken, M.F. Nico. J. Phys.: Conf. Ser., 73 (9), 1154 (2012).

[17] A.J. Minnich, M.S. Dresselhaus, Z.F. Ren, G. Chen. Energy Environ. Sci., 2, 466 (2009).

\section{Effective mass, charge carrier mobility, and lattice thermal conductivity in nanocomposite thermoelectrics based on bismuth and antimony chalcogenides}

\section{L.N. Lukyanova, A.A. Shabaldin, A.Yu. Samunun, O.A. Usov}

loffe Institute, 194021 St. Petersburg, Russia

\begin{abstract}
In the p-type thermoelectrics based on bismuth chalcogenides and antimony with an excess bismuth, the density of states effective mass $\mathrm{m} / \mathrm{m}_{0}$ increases in nanocomposite and nanostructured solid solutions compared with the base material obtained by the direct crystallization method. It is shown that an increase in $m / m_{0}$ is associated with an increase in the effective scattering parameter $r_{\text {eff }}$ and amplifying the relaxation time of energy, which is typical for topological insulators. The material parameter $\beta$, proportional to the thermoelectric efficiency of $Z T$, at temperatures below room increases stronger in nanostructured composition than in a nanocomposite with the inclusions of $\mathrm{SiO}_{2}$ due to the growth of $m / m_{0}$ and decrease the lattice thermal conductivity $\kappa_{\mathrm{L}}$. At high temperatures in the range of 300-500 to the greatest growth of the parameter $\beta$ in the base material associated with higher mobility.
\end{abstract}

